\title{
Effect of the length of the tool pin on the hardening of 2024 aluminum alloy under friction stir processing
}

\author{
A. Kh. Valeeva ${ }^{\dagger, 1,2}$, A. Kh. Akhunova ${ }^{1,2}$, D. B. Kabirova ${ }^{1}$, M. F. Imayev ${ }^{1,2}$, R.F. Fazlyakhmetov ${ }^{1,2}$ \\ †valeevs@mail.ru
}

${ }^{1}$ Institute for Metals Superplasticity Problems, RAS, Ufa, 450001, Russia

${ }^{2}$ Bashkir State University, Ufa, 450076, Russia

The paper reports the results of studies on the effect of the tool pin length on the microstructure and hardness of 2024 aluminum alloy sheets with a thickness of $3.0 \mathrm{~mm}$ during friction stir processing (FSP), as well as computer simulation data. The alloy in the initial state contains particles of phases $\theta\left(\mathrm{Al}_{2} \mathrm{Cu}\right), S\left(\mathrm{Al}_{2} \mathrm{CuMg}\right)$, and phases of complex composition $(\mathrm{AlCuMnSiFe})$ with sizes ranging from $0.5-3$ to several tens of microns. There is also a small amount of silicon-rich particles with sizes of $2-3 \mu \mathrm{m}$. The phases (AlCuMnSiFe) in their initial state often form skeletal precipitates up to $30-40 \mu \mathrm{m}$ in size. Such precipitates consist of three phases differing in the ratio of elements. FSP of the material led to an increase in microhardness in the center of the treated zone and the advancing side from $53 \pm 4 \mathrm{HV}$ to $110 \pm 8 \mathrm{HV}$, while the highest microhardness values practically do not depend on the pin length. The distribution of microhardness and microstructure in the processing area is uneven and depends on the length of the tool pin. The highest hardness of the central FSP zone is provided by a pin $2.0 \mathrm{~mm}$ long. It has been established that during the FSP of the alloy, coarse particles of intermetallic phases are refined and the products of refining in the FSP zone are distributed. The particles of the $\theta$ and $S$ phases are refined weakly, while the others are refined to submicron sizes. To substantiate the choice of the effective pin length, a three-dimensional finite element modeling of the FSP in the DEFORM-3D software environment was performed. Distributions of effective deformation, temperature fields, and displacement of material points in the zone of thermomechanical action are analyzed. The simulation results agree with data of the physical experiment in that the most preferable pin under the considered processing conditions is a pin with a length of $2.0 \mathrm{~mm}$ since it allows one to obtain the widest and most symmetrical regions of intense heating and deformation.

Keywords: friction stir processing, hardening, computer modeling, DEFORM-3D.

УДК: 621.791 .14

\section{Влияние длины наконечника инструмента на упрочнение алюминиевого сплава Д16 при обработке трением с перемешиванием}

\author{
Валеева А. Х. ${ }^{\dagger, 1}$, Ахунова А. Х. ${ }^{1,2}$, Кабирова Д. Б. ${ }^{1}$, \\ Имаев М. Ф. ${ }^{1,2}$, Фазлыахметов Р..$^{1,2}$ \\ ${ }^{1}$ Институт проблем сверхпластичности металлов РАН, Уфа, 450001, Россия \\ ${ }^{2}$ Башкирский государственный университет, Уфа, 450076, Россия
}

Представлены результаты исследования влияния длины наконечника инструмента на микроструктуру и твердость
листа алюминиевого сплава Д16 толщиной 3.0 мм при обработке трением с перемешиванием (OTП), а также
данные компьютерного моделирования. Сплав в исходном состоянии содержит частицы фаз $\theta\left(\mathrm{Al}_{2} \mathrm{Cu}\right), S\left(\mathrm{Al} \mathrm{CuMg}_{2} \mathrm{M}\right.$ )
и фаз сложного состава (AlCuMnSiFe) размером от $0.5-3$ до нескольких десятков микрон. Также наблюдается
небольшое количество частиц размером $2-3$ мкм, богатых кремнием. Фазы (AlCuMnSiFe) в исходном состоянии
часто образуют выделения скелетообразной формы размером до $30-40$ мкм. Такие выделения состоят из трех
фаз, различающихся соотношением элементов. ОTП материала привела к росту микротвердости в центре обра-
батываемой зоны и в стороне набегания с $53 \pm 4 \mathrm{HV}$ до $110 \pm 8$ НV, при этом наибольшие значения микротвердости
практически не зависят от длины наконечника. Распределение микротвердости и микроструктуры в зоне обработки
неравномерное и зависит от длины наконечника инструмента. Наиболее высокую твердость центральной зоны
ОТП обеспечивает наконечник длиной 2.0 мм. Установлено, что при ОТП сплава происходит измельчение крупных 
частиц интерметаллидных фаз и распределение продуктов измельчения в зоне ОТП. Частицы фаз $\theta$ и $S$ слабо подвержены измельчению, в то время как остальные фазы измельчаются вплоть до субмикронного размера. Для обоснования выбора эффективной длины наконечника выполнено трехмерное конечно-элементное моделирование процесса обработки методом ОTП в программной среде DEFORM-3D. Проанализировано распределения эффективной деформации, температурных полей и перемещения материальных точек в зоне термомеханического воздействия. Результаты моделирования согласуются с данными физического эксперимента в том, что наиболее предпочтительным при рассмотренных условиях обработки является наконечник длиной 2.0 мм, так как он позволяет получить наиболее широкие и симметричные области интенсивного разогрева и деформации.

Ключевые слова: обработка трением с перемешиванием, упрочнение, компьютерное моделирование, DEFORM-3D.

\section{1. Введение}

Сварку трением с перемешиванием (СТП) применяют в основном для соединения материалов со сравнительно низкой температурой плавления, прежде всего алюминиевых сплавов [1-4]. Согласно данной технологии, сварку листовых заготовок производят с помощью сил трения, создаваемого инструментом, состоящим из заплечика и наконечника. Инструмент, вращающийся с высокой скоростью, перемещают вдоль линии соединения двух жестко закреплённых заготовок. Аналогичной процессу СТП является перемешивающая фрикционная обработка или обработка трением с перемешиванием (ОТП). Единственное отличие заключается в том, что при ОТП не происходит соединения двух заготовок металла, заготовка изначально является монолитной. В результате ОТП происходит измельчение зеренной структуры и повышение комплекса механических свойств металлического материала $[4,5]$.

Известно несколько основных технологий получения наноструктурированных материалов, основанных на наномодификации металлических расплавов, методах порошковой металлургии и интенсивной пластической деформации $[6,7]$. Для получения объемных заготовок с ультрамелкозернистой или наноструктурой наиболее часто применяют методы интенсивной пластической деформации. Обработку трением с перемешиванием также можно отнести к методам интенсивной пластической деформации $[8,9]$, так как она обеспечивает высокие интенсивности деформации.

В последнее время технологию обработки трением с перемешиванием используют для модификации микроструктуры, гомогенизации и снижения пористости, для улучшения механических свойств различных металлов и полимеров [9]. В результате ОТП часто формируется сильно измельченная зеренная микроструктура [10], а многопроходная ОТП литых металлов приводит к преобразованию литой микроструктуры в кованую без изменения формы заготовки [10-11]. В [12,13] отмечается, что ОТП высокопрочных низколегированных сталей приводит к улучшению микроструктуры и значительному увеличению твердости и прочности (на 30 и 34\%, соответственно). В связи с этим можно предположить, что использование данного метода позволит существенно измельчить микроструктуру и повысить комплекс механических свойств металлических материалов.

Результат ОТП зависит, в частности, от конструкции инструмента, которая влияет на тепловыделение, течение материала, усилия и другие выходные характеристики [8-13]. Для выбора эффективных параметров процесса ОТП целесообразно использовать компьютерное моделирование, что позволяет существенно снизить затраты на изготовление дорогостоящего специального инструмента [14-16].

Целью данной работы было определить зависимость микроструктуры и твердости алюминиевого сплава Д16 от глубины погружения наконечника инструмента в листовую заготовку при ОТП, а также провести моделирование распределения температуры, эффективной деформации и перемещения материальных точек в зоне воздействия инструмента в зависимости от длины наконечника.

\section{2. Методики эксперимента}

В качестве материала исследования был выбран промышленный деформируемый термоупрочняемый алюминиевый сплав Д16 в виде листов толщиной 3.0 мм. Для проведения ОТП использовали инструмент, модель которого показана на Рис. 1. Выдвигаемый наконечник диаметром 6 мм имеет левую резьбу. Диаметр заплечика 16 мм и на нем имеются два спиралеобразных выступа. Длину наконечника варьировали от 1.6 до 2.2 мм. Обработку проводили при скорости перемещения инструмента $v=3 \mathrm{~cm} /$ мин, частоте вращения $\omega=1000$ об/мин и осевом усилии $P=2$ кН.

Микротвердость определяли по методу Виккерса на цифровом микротвердомере MVDM 8 «AFFRY» при нагрузке $0.5 \mathrm{H}$ и длительности ее приложения 10 секунд. Измерения проводили на образцах шириной 22.0 и толщиной 3.0 мм, вырезанных перпендикулярно направлению поступательного движения вращающегося

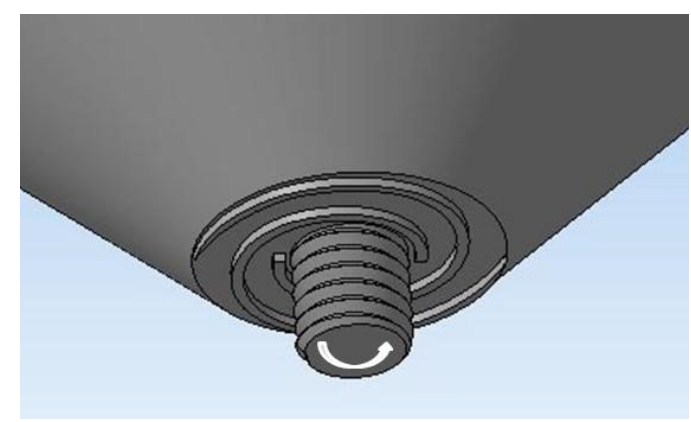

Рис. 1. Модель инструмента для ОТП. Направление вращения наконечника показано стрелкой.

Fig. 1. Model of the tool for FSP. The arrow shows the pin rotation direction. 
инструмента. Микротвердость определяли в точках пересечения воображаемых прямых, проведенных на расстоянии $0.6,1.1,1.6,2.1,2.6$ мм от поверхности образца, и на расстоянии $5,8,11,14,17$ мм по ширине образца. Ошибка измерения микротвердости не превышала 5\%.

Процессы тепловыделения в образце изучали при моделировании в среде программного продукта DEFORM-3D. Рассматривали лист из алюминиевого сплава Д16 толщиной 3.0 мм, длиной и шириной 40.0 и 30.0 мм, соответственно. Моделировали обработку при скорости перемещения инструмента $3 \mathrm{~cm} / \mathrm{мин,} \mathrm{частоте} \mathrm{вращения}$ 1000 об/мин и осевом усилии 2 кН. Конечно-элементная модель листа состояла из 60000 элементов, а инструмента - из 32000 (более подробно описана в [17]).

Поведение материала листа описывали с помощью модели Джонсона-Кука, входящей в стандартную библиотеку DEFORM-3D. Задавали следующие физические постоянные сплава: плотность - 2640 кг/м³ теплопроводность - $122 \mathrm{BT} /(\mathrm{M} \cdot \mathrm{K})$; теплоемкость при постоянном давлении - 922 Дж/(кг·К). При обработке трением с перемешиванием листовой заготовке задавали условие теплообмена с окружающей средой. Начальная температура листа, инструмента и окружающей среды составляла $20^{\circ} \mathrm{C}$. Со всех внешних поверхностей была задана конвекция с коэффициентом теплопередачи $11 \mathrm{~B} /\left(\mathrm{M}^{2} \cdot\right.$ К). В целях уменьшения погрешности расчета теплофизические свойства сплава Д16 были приняты постоянными и равными значениям, достигаемым в материале при температуре $600^{\circ} \mathrm{C}$. Инструмент принимали абсолютно жестким телом. В качестве материала инструмента выбрали инструментальную сталь AISI-D2. Геометрия наконечника инструмента была упрощена для уменьшения времени расчетов. Рассмотрели наконечники в форме цилиндра с разной длиной: 1.6, 1.8, 2.0, 2.2 мм. Диаметр нижней части наконечника составлял 2 мм. Коэффициент трения принимали равным 0.5.

Для выявления структуры в зоне термомеханического воздействия образцы сначала подвергали механической полировке, затем - химическому травлению реактивом Келлера [18]. Исследование микроструктуры проводили на световом микроскопе "Axiovert-100A» и растровом электронном микроскопе TESCAN VEGA3, снабженном приставкой для энергодисперсионного анализа Oxford Instruments X-act.

\section{3. Результаты и обсуждение}

\section{1. Микротвердость в обрабатываемой зоне}

Результаты измерения микротвердости приведены на Рис. 2. Обработка материала перемешиванием привела к существенному росту микротвердости в центре обрабатываемой зоны и в стороне набегания с $53 \pm 4 \mathrm{HV}$ до $110 \pm 8 \mathrm{HV}$.

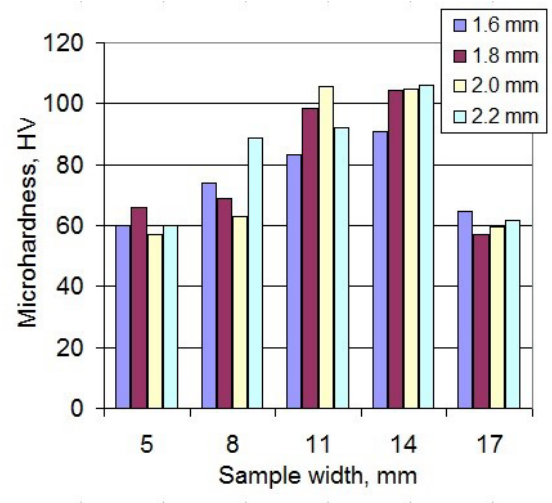

a

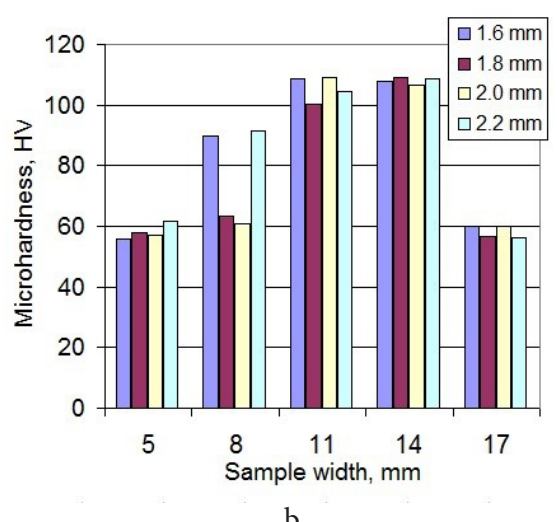

b

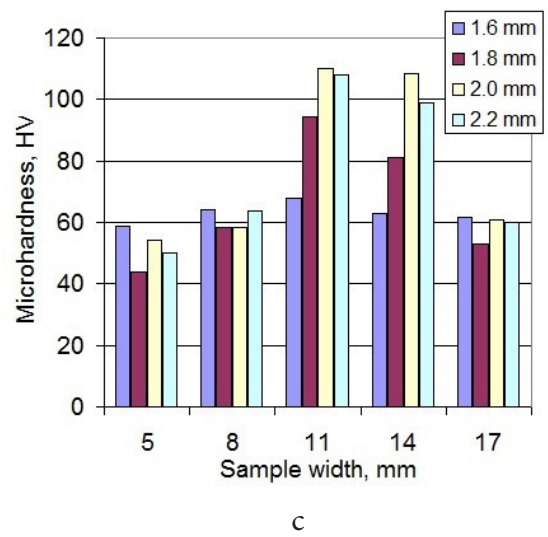

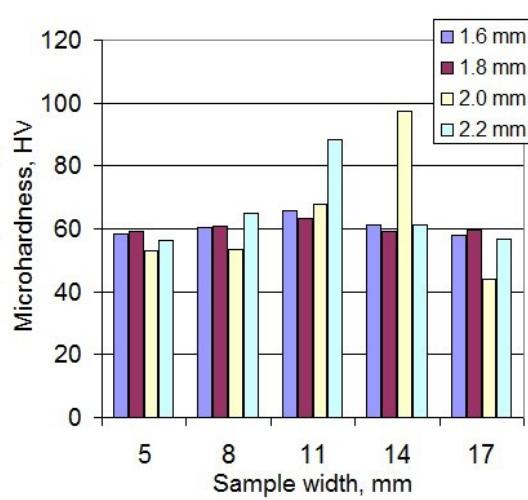

d

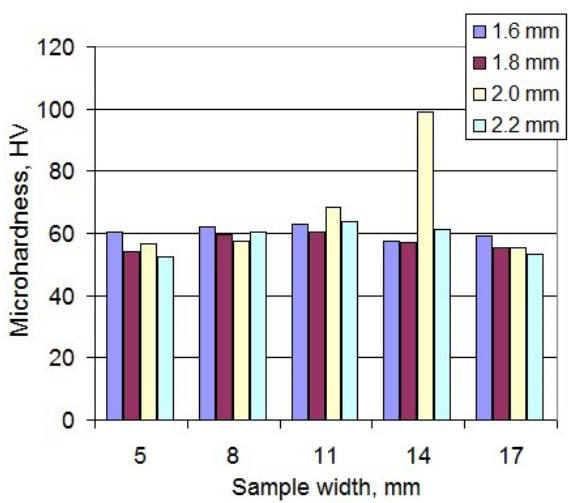

Pис. 2. (Color online) Микротвердость слоев образца, находящихся на глубине 0.6 мм (a), 1.1 мм (b), 1.6 мм (c), 2.1 мм (d), 2.6 мм (e) от поверхности после ОТП наконечниками различной длины. Сторона набегания расположена справа.

Fig. 2. (Color online) Microhardness of sample layers located at a depth of $0.6 \mathrm{~mm}$ (a), $1.1 \mathrm{~mm}$ (b), $1.6 \mathrm{~mm}$ (c), $2.1 \mathrm{~mm}$ (d), $2.6 \mathrm{~mm}$ (e) from the surface after FSP with pins of various lengths. The advancing side is located on the right. 
На глубинах 0.6 и 1.1 мм повышение микротвердости в зоне термомеханического воздействия обеспечивают все рассмотренные наконечники (Рис. $2 \mathrm{a}, \mathrm{b})$. Наконечника длиной 1.6 мм оказывается недостаточно для упрочнения центральной части обрабатываемой зоны на глубине 1.6 мм (Рис. 2c). На глубине 2.1 мм только наконечники длиной 2.0 и 2.2 мм приводят к росту микротвердости (Рис. $2 \mathrm{~d}$ ). Наибольшее упрочнение на глубине 2.6 мм обеспечивает наконечник длиной 2.0 мм. В целом, только обработка наконечником длиной 2.0 мм обеспечивает упрочнение всех рассмотренных слоев образца. Следует отметить, что наиболее сильное (двукратное) упрочнение имеет место на стороне набегания, при этом наибольшие значения микротвердости практически не зависят от длины наконечника. На основании проведенных исследований микротвердости образцов толщиной 3.0 мм можно заключить, что необходимой и достаточной длиной наконечника для увеличения механических свойств в зоне термомеханического воздействия является 2.0 мм.

\section{2. Микроструктура}

\subsection{1. Исходная микроструктура}

В исходном состоянии сплава в равновесии с алюминиевой матрицей - $\alpha$-твердым раствором меди и магния в алюминии - находятся фазы $\theta\left(\mathrm{Al}_{2} \mathrm{Cu}\right)$, $S\left(\mathrm{Al}_{2} \mathrm{CuMg}\right)[19]$ и фазы сложного состава, содержащие алюминий, медь, марганец, кремний и железо (AlCuMnSiFe). Размер частиц этих фаз варьируется от 0.5-3 до нескольких десятков микрон. Также наблюдается небольшое количество частиц размером 2-3 мкм богатых кремнием. Возможно, эти частицы представляют собой чистый кремний, т. к. содержание $\mathrm{Si}$ в них превышает $40 \%$. Фазы (AlCuMnSiFe) в исходном состоянии часто образуют выделения скелетообразной формы, достигающие в размере 30-40 мкм (Рис. 3). В представленном выделении обнаруживаются три фазы: серого цвета, белого цвета, а также мелкие вкрапления ярко белого цвета (Рис. 3, режим BSE).

Карта распределения элементов (Рис. 4) показывает, что серая фаза обогащена $\mathrm{Fe}, \mathrm{Mn}$ и $\mathrm{Si}$, в то время как,

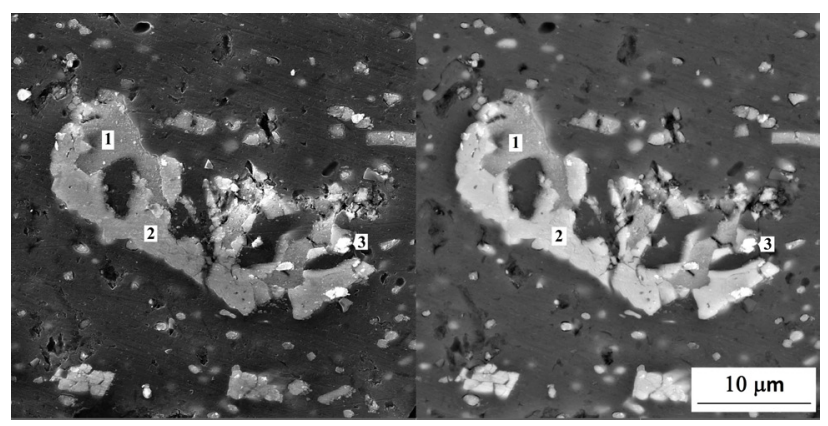

Рис. 3. Скелетообразное выделение в исходном состоянии: режим SE (слева), BSE (справа). 1 - серая фаза, 2 - белая фаза, 3 - ярко белая фаза.

Fig. 3. Skeletal precipitation in initial state: SE mode (left), BSE (right). 1 - gray phase, 2 - white phase, 3 - bright white phase. белая фаза содержит больше $\mathrm{Cu}$, но обеднена $\mathrm{Fe}$ и $\mathrm{Mn}$ и содержит очень мало Si. Ярко белая фаза отличается повышенным содержанием Cu. Составы описанных выше фаз приведены в Табл. 1. Подобные скелетообразные выделения в сплаве Д16 наблюдали в работе [20].

\subsection{2. Микроструктура зонь перемешивания после ОТП}

Исследование микроструктуры методом световой микроскопии позволило увидеть на поверхности травленых шлифов ярко выраженные полосы, так называемые «луковые кольца», а также зону термомеханического воздействия [21]. На Рис. 5 представлена микроструктура центра зоны перемешивания, наблюдаемая в РЭМ. Видны два «луковых кольца», граница между которыми отмечена стрелками.

Нижнее «луковое кольцо» содержит высокую плотность субмикронных частиц, в то время как в верхнем

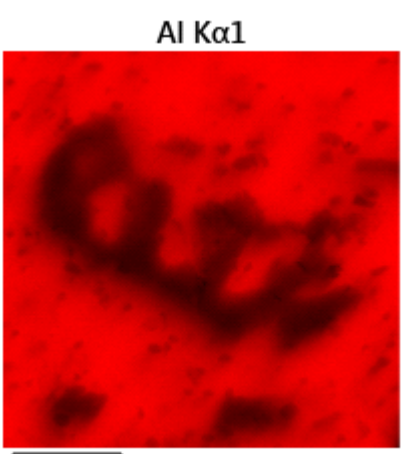

$10 \mu \mathrm{m}$

$10 \mu \mathrm{m}$

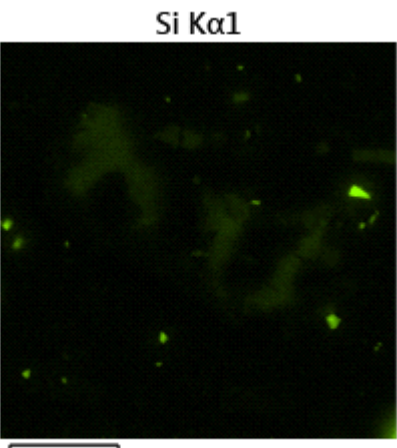

$10 \mu \mathrm{m}$

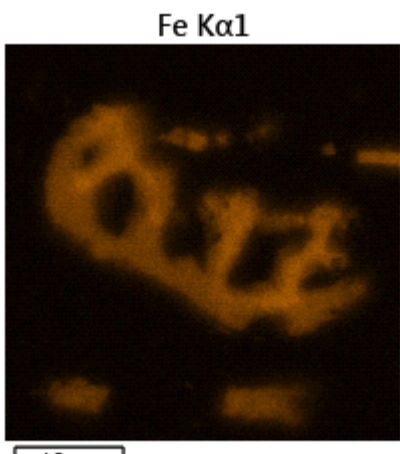

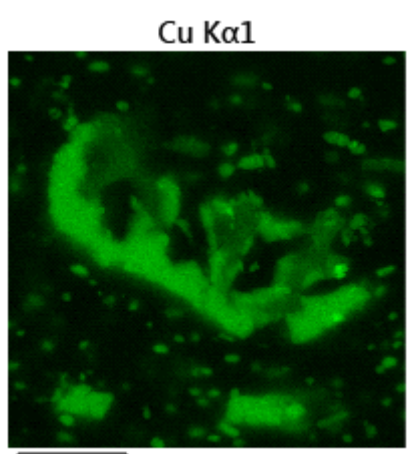

$10 \mu \mathrm{m}$

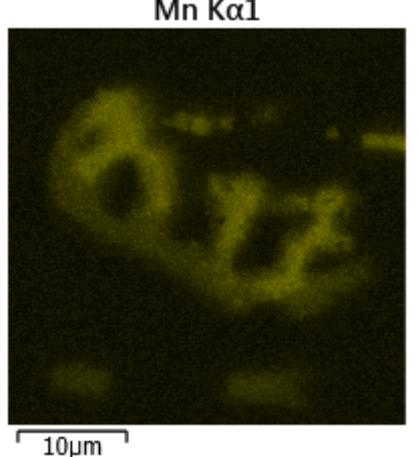

$\mathrm{Mg} \mathrm{K \alpha 1 \_ 2}$

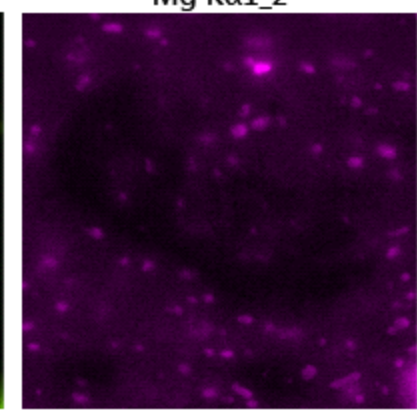

$10 \mu \mathrm{m}$

Pис. 4. (Color online) Карты распределения элементов в скелетообразном выделении.

Fig. 4. (Color online) Element distribution maps in the skeletal precipitation. 
Табл. 1. Составы фаз, входящих в скелетообразное выделение, ат.\%.

Table 1. Compositions of the phases included in the skeletal precipitation, at.\%.

\begin{tabular}{|c|c|c|c|c|c|}
\hline Фаза / Phase & $\mathrm{Cu}$ & $\mathrm{Si}$ & $\mathrm{Fe}$ & $\mathrm{Mn}$ & $\mathrm{Al}$ \\
\hline Серая / Grey & 6 & 5 & 8 & 3 & 77 \\
\hline Белая / White & 17 & 1 & 7 & 2 & 73 \\
\hline Ярко белая / Bright white & 18 & 3 & 5 & 2 & 72 \\
\hline
\end{tabular}

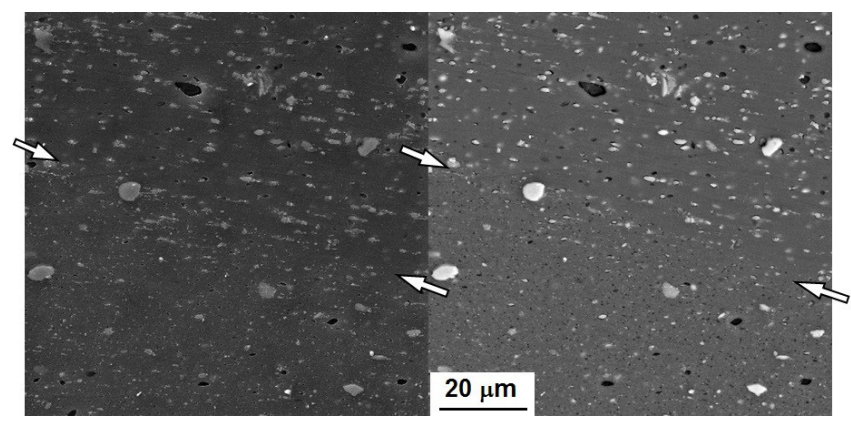

Рис. 5. Микроструктура зоны «луковых колец». Стрелками показана граница, ниже которой располагается область с высокой плотностью субмикронных частиц. Режим SE (слева), BSE (справа).

Fig. 5. Microstructure of the "onion rings" zone. The arrows show the boundary below which the region with a high density of submicron particles is located. SE mode (left), BSE (right).

«луковом кольце» такие частицы практически отсутствуют. В зоне перемешивания наблюдаются фазы $\theta$ и $S$ размером до 10 мкм. Кроме того, в зоне перемешивания наблюдаются частицы двух фаз, отличающихся соотношением элементов $\mathrm{Al}, \mathrm{Cu}, \mathrm{Mn}, \mathrm{Si}$ и $\mathrm{Fe}$. Одна фаза близка по составу к известной фазе (CuFeMn) ${ }_{3} \mathrm{Si}_{2} \mathrm{Al}_{15}$, другая - ближе к ( $\mathrm{SiFeMn}) \mathrm{Cu}_{2} \mathrm{Al}_{6}$ [22]. В незначительном количестве наблюдается также фаза (AlCuFe). Стоит отметить, что фазы $\theta$ и $S$ слабо подвержены измельчению, в то время как остальные фазы измельчаются вплоть до субмикронного размера.

Таким образом, интенсивная деформация перемешиванием разогретого до высоких температур материала вызывает фрагментацию и распределение интерметаллидных частиц в зоне обработки материала, что приводит к повышению ее твердости.

\section{3. Компьютерное моделирование}

На Рис. 6 показаны типовые эпюры распределения температуры, эффективной деформации и перемещения материальных точек в поперечном сечении обрабатываемого листа в зависимости от длины наконечника. Видно, что в процессе ОТП формируются неравномерные поля распределения температуры в обрабатываемой области в поперечном направлении листа (Рис. 6а), при этом наибольшее значение температуры наблюдается на стороне набегания. Также следует отметить, что по мере удлинения наконечника увеличивается и максимальная температура разогрева листа. Наиболее обширная и достаточно симметричная зона термомеханического воздействия с температурой в интервале $383-455^{\circ} \mathrm{C}$ формируется при длине наконечника 2.0 мм. При длине наконечника 2.2 мм область, разогретая до $T=383-455^{\circ} \mathrm{C}$, уменьшается и смещается в сторону набегания, ближе к нижней части листа. Согласно работе [23] наиболее качественная проработка материала листа происходит при температурах около $0.8 T_{\text {пл, }}$ т. к. разогрев до таких температур обеспечивает высокую пластичность и хорошее перемешивание материала. Для сплава Д16 величина $0.8 T_{\text {пл }}$ в зависимости от вариаций состава составляет $385-425^{\circ} \mathrm{C}$. Область высоких значений эффективных деформаций (43.8-50 мм/мм) увеличивается с ростом длины наконечника и смещается к нижней части листа (Рис. 6b). Наиболее широкая и симметричная область эффективных деформаций на уровне 43.8-50 мм/мм наблюдается при наконечнике длиной 2.0 мм.

Результаты моделирования показывают, что материал в нижней части листа практически не подвергается перемешиванию, и чем меньше длина наконечника, тем эта область больше. В основном перемешиванию подвергаются области в средней и верхней части листа, протяженность прорабатываемых областей растет с увеличением длины наконечника. При этом по эпюрам распределения перемещения материальных точек (Рис. 6с) следует, что материал листа со стороны набегания в меньшей степени участвует в перемешивании, оставаясь в основном в своей области, материал же с отступающей стороны активно переходит на сторону набегания и смешивается с материалом из области

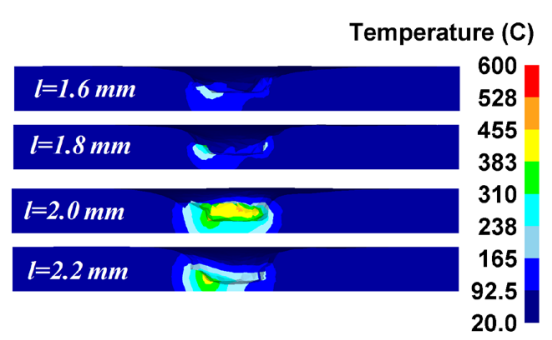

a

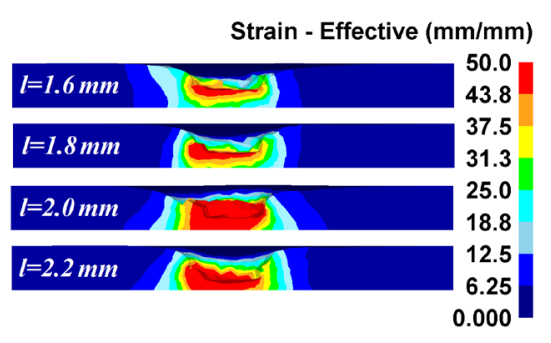

b

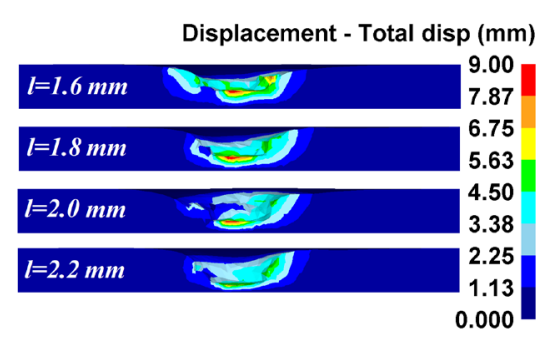

Pис. 6. (Color online) Распределения температуры (a), эффективной деформации (b) и перемещения материальных точек (c) в зависимости от длины наконечника в поперечном сечении листа в процессе обработки трением с перемешиванием. Сторона набегания слева.

Fig. 6. (Color online) Distribution of temperature (a), effective strain (b), and displacement of material points (c) depending on the length of the pin in the cross-section of the sheet during friction stir processing. The advancing side is on the left. 
набегания. Такая закономерность наблюдается для всех длин наконечника.

Таким образом, результаты компьютерного моделирования согласуются с данными эксперимента: для увеличения прочностных характеристик алюминиевого сплава Д16 в зоне термомеханического воздействия необходимой и достаточной длиной наконечника является 2.0 мм.

\section{4. Выводы}

1. По результатам эксперимента и компьютерного моделирования установлено, что из всех рассмотренных наконечников предпочтительным для ОТП алюминиевого сплава Д16 при данных условиях является наконечник длиной 2.0 мм, обеспечивающий формирование широких и симметричных областей термомеханического воздействия с благоприятной температурой около $0.8 T_{\text {пл }}$ и наиболее однородной областью высоких значений эффективной деформации.

2. При обработке трением с перемешиванием алюминиевого сплава Д16 происходит дисперсионное упрочнение зоны термомеханического воздействия, связанное с перераспределением и фрагментацией частиц интерметаллидных фаз.

Благодарности / Acknowledgments. Работа выполнена в рамках государственного задания ИПСМ РАН № AAAA-A19-119021390106-1. / The present work was accomplished according to the state assignment of IMSP RAS No. AAAA-A19-119021390106-1.

\section{Литература/References}

1. S. Mironov, Y.S. Sato, H. Kokawa. J. of Mater. Sci. and Technol. 34, 58 (2018). Crossref

2. H. J. Liu, J. J. Shen, Y. X. Huang, L. Y. Kuang, C. Liu, C. Li. Sci. Technol. Weld. Joining. 14 (6), 577 (2009). Crossref

3. M. Sarvghad Moghaddam, R. Parvizi, M. HaddadSabzevar, A. Davoodi. Materials and Design. 32 (5), 2749 (2011). Crossref

4. P. Xue, G. M. Xie, B.L. Xiao, Z. Y. Ma, L. Geng. Metall. Mater. Trans. A. 41, 2010 (2010). Crossref

5. A.I. Rudskoy, A. A. Naumov, E. V. Chernikov. Tsvetnyye metally. Chernyye metally. Special issue, 24. (2014). (in Russian) [А. И. Рудской, А. А. Наумов, Е. В. Черников. Цветные металлы. Черные металлы. Спец. вып., 24 (2014).]

6. R.Z. Valiev, I. V. Alexandrov. Nanostructured materials obtained by severe plastic deformation. Moscow, Logos (2000) 272 p. (in Russian) [Р.3. Валиев, И.В. Александров. Наноструктурные материалы, полученные интенсивной пластической деформацией. Москва, Логос (2000) 272 с.]
7. A.I. Rudskoy. Nanostrukturirovannye metallicheskie materialy (Nanostructured metallic materials). Nauka, S.-Petersburg (2011) 270 p. (in Russian) [А. И. Рудской. Наноструктурированные металлические материалы. Наука, С.-Петербург (2011) 270 с.]

8. H. Pashazadeh, J. Teimournezhad, A. Masoumi. Materials and Design. 55, 619 (2014). Crossref

9. M.K. Besharati-Givi, Parviz Asadi. Advances in Friction Stir Welding and Processing. Elsevier, Woodhead Publishing (2014) 823 p.

10. T. R. McNelley. Lett. Mater. 5 (3), 246 (2015). Crossref

11. S. M. Aktarer, T. Küçükömeroğlu. Journal of Achievements of Materials and Manufacturing Engineering. 75 (2), 55 (2016). $\underline{\text { Crossref }}$

12. Y. Chen, H. Ding, J. Li, Z. Cai, J. Zhao, W. Yang. Mater. Sci. Eng. A. 650, 281 (2016). Crossref

13. X. Chen, Y. Zhang, M. Cong. Vacuum. 175, 109292 (2020). $\underline{\text { Crossref }}$

14. M. Elyasi, H. A. Derazkola, M. Hosseinzadeh. Proceedings of the Institution of Mechanical Engineers, Part B: J. of Engineering Manufacture. 230, 1234 (2016). $\underline{\text { Crossref }}$

15. M. Assidi, L. Fourment, S. Guerdoux, T. Nelson. Int. J. of Mach. Tools and Manuf. 50 (2), 143 (2010). Crossref

16. T. Nakamura, T. Obikawa, E. Yukutake, S. Ueda, I. Nishizaki. Modern Mechanical Engineering. 8 (1), 78 (2018). Crossref

17. A. K. Akhunova, M. F. Imayev, A. K. Valeeva. Lett. Mater. 9 (4), 456 (2019). Crossref

18. V.S. Kovalenko. Metallurgical reagents. Moscow, Metallurgiya (1981) 120 p. (in Russian) [В. С. Коваленко. Металлографические реактивы. Москва, Металлургия (1981) 120 с.]

19. L.F. Mondolfo. Structure and Properties of Aluminum Alloys. Moscow, Metallurgy (1979) 640 p. (in Russian) [Л.Ф. Мондольфо. Структура и свойства алюминиевых сплавов. Москва, Металлургия (1979) 640 c.]

20. A. Staszczyk, J. Sawicki, B. Adamczyk-Cieslak. Materials (Basel). 12 (24), 4168 (2019). Crossref

21. A.Kh. Valeeva, A.Kh. Akhunova, M.F. Imayev, R.F.Fazlyakhmetov.Ultrafine-grainedand nanostructured materials: Proceedings of the Open School-Conference of the CIS Countries. Ufa, RIC BashSU (2020) p. 199. (in Russian) [А.Х. Валеева, А.Х. Ахунова, М.Ф. Имаев, Р.Ф. Фазлыахметов. Ультрамелкозернистые и наноструктурные материалы: Сборник трудов Открытой школы-конференции стран СНГ. Уфа, РИЦ БашГУ (2020) c. 199.]

22. R. G. Buchheit, R.P. Grant, P.F. Hiava, B. Mckenzie, G. L. Zender. J. of the Electrochemical Society. 144 (8), 2621 (1997). Crossref

23. R. S. Mishra, Z. Y. Ma. Mater. Sci. and Eng.: R. 50(1-2), 1 (2005). rossref 